\title{
RELATIVE POSITION OF THREE SUBSPACES IN A HILBERT SPACE
}

\author{
MASATOSHI ENOMOTO AND YASUO WATATANI
}

\begin{abstract}
We study the relative position of three subspaces in a separable infinite-dimensional Hilbert space. In the finite-dimensional case, Brenner described the general position of three subspaces completely. We extend it to a certain class of three subspaces in an infinite-dimensional Hilbert space. We also give a partial result which gives a condition on a system to have a (dense) decomposition containing a pentagon.
\end{abstract}

KEYWORDS: three subspaces, Hilbert space,

AMS SUBJECT CLASSIFICATION: 46C07, 47A15, 15A21, 16G20, $16 \mathrm{G} 60$.

\section{INTRODUCTION}

We study the relative position of three subspaces in a separable infinite-dimensional Hilbert space.

The relative position of one subspace of a Hilbert space is extremely simple and determined by the dimension and the co-dimension of the subspace. It is a well known fact that the relative position of two subspaces $E$ and $F$ in a Hilbert space $H$ can be described completely up to unitary equivalence as in Araki [1, Davis [4, Dixmier [5] and Halmos [15]. The Hilbert space is the direct sum of five subspaces:

$$
H=(E \cap F) \oplus(\text { the rest }) \oplus\left(E \cap F^{\perp}\right) \oplus\left(E^{\perp} \cap F\right) \oplus\left(E^{\perp} \cap F^{\perp}\right) .
$$

In the rest part, $E$ and $F$ are in generic position and the relative position is described only by "the angles" between them.

We disregard "the angles" and study the still-remaining fundamental feature of the relative position of subspaces. This is the reason why we use bounded invertible operators instead of unitaries to define isomorphisms in our paper.

Let $H$ be a Hilbert space and $E_{1}, \ldots E_{n}$ be $n$ subspaces in $H$. Then we say that $\mathcal{S}=\left(H ; E_{1}, \ldots, E_{n}\right)$ is a system of $n$ subspaces in $H$ or an $n$-subspace system in $H$. Let $\mathcal{T}=\left(K ; F_{1}, \ldots, F_{n}\right)$ be another system of $n$-subspaces in a Hilbert space $K$. We say that systems $\mathcal{S}$ and $\mathcal{T}$ are isomorphic if there is a bounded invertible operator $\varphi: H \rightarrow K$ satisfying that $\varphi\left(E_{i}\right)=F_{i}$ for $i=1, \ldots, n$. See also Sunder [24] for other topics on $n$-subspaces. 
In [3] S. Brenner gave a complete description of systems of three subspaces up to isomorphims when an ambient space $H$ is finite-dimensional.

A system $\mathcal{S}$ is called indecomposable if $\mathcal{S}$ can not be decomposed into a nontrivial direct sum. If the ambient Hilbert space $H$ is finitedimensional, then any system of $n$ subspaces in $H$ is a finite direct sum of indecomposable systems.

Let $\mathcal{S}=\left(H ; E_{1}, E_{2}, E_{3}\right)$ be an indecomposable system of three subspaces in a finite-dimensional Hilbert space $H$. Then $\mathcal{S}$ is isomorphic to one of the following eight trivial systems $\mathcal{S}_{1}, \ldots, \mathcal{S}_{8}$ and one non-trivial system $\mathcal{S}_{9}$ :

$$
\begin{gathered}
\mathcal{S}_{1}=(\mathbb{C} ; 0,0,0), \quad \mathcal{S}_{2}=(\mathbb{C} ; \mathbb{C}, 0,0), \quad \mathcal{S}_{3}=(\mathbb{C} ; 0, \mathbb{C}, 0), \\
\mathcal{S}_{4}=(\mathbb{C} ; 0,0, \mathbb{C}), \quad \mathcal{S}_{5}=(\mathbb{C} ; \mathbb{C}, \mathbb{C}, 0), \quad \mathcal{S}_{6}=(\mathbb{C} ; \mathbb{C}, 0, \mathbb{C}), \\
\mathcal{S}_{7}=(\mathbb{C} ; 0, \mathbb{C}, \mathbb{C}), \mathcal{S}_{8}=(\mathbb{C} ; \mathbb{C}, \mathbb{C}, \mathbb{C}), \mathcal{S}_{9}=\left(\mathbb{C}^{2} ; \mathbb{C}(1,0), \mathbb{C}(0,1), \mathbb{C}(1,1)\right) .
\end{gathered}
$$

See, for example, [10], [14] or [6] on indecomposable systems of $n$ subspaces.

Therefore we have the following theorem of Brenner:

Theorem 1.1 (Brenner [3]). Let $\mathcal{S}=\left(H ; E_{1}, E_{2}, E_{3}\right)$ be a system of three subspaces in a finite-dimensional Hilbert space $H$. Then $\mathcal{S}$ is isomorphic to the following $\mathcal{T}=\left(H ; F_{1}, F_{2}, F_{3}\right)$ such that there exist subspaces $S, N_{1}, N_{2}, N_{3}, M_{1}, M_{2}, M_{3}, Q, L$ of $H$ satisfying that $Q$ has a form

$$
\left(Q ; Q_{1}, Q_{2}, Q_{3}\right):=(K \oplus K ; K \oplus 0,0 \oplus K,\{(x, x) \mid x \in K\})
$$

of double triangle and

$$
\begin{aligned}
& H=S \oplus N_{1} \oplus N_{2} \oplus N_{3} \oplus M_{1} \oplus M_{2} \oplus M_{3} \oplus Q \oplus L \\
& F_{1}=S \oplus 0 \quad \oplus N_{2} \oplus N_{3} \oplus M_{1} \oplus 0 \quad \oplus 0 \quad \oplus Q_{1} \oplus 0 \\
& F_{2}=S \oplus N_{1} \oplus 0 \quad \oplus N_{3} \oplus 0 \quad \oplus M_{2} \oplus 0 \quad \oplus Q_{2} \oplus 0 \\
& F_{3}=S \oplus N_{1} \oplus N_{2} \oplus 0 \quad \oplus 0 \quad \oplus 0 \quad \oplus M_{3} \oplus Q_{3} \oplus 0
\end{aligned}
$$

Remark. In the above decomposition, we can choose $\mathcal{T}$ such that $S=F_{1} \cap F_{2} \cap F_{3}, N_{1}=F_{1}^{\perp} \cap F_{2} \cap F_{3}, N_{2}=F_{1} \cap F_{2}^{\perp} \cap F_{3}, N_{3}=F_{1} \cap F_{2} \cap F_{3}^{\perp}$, $M_{1}=F_{1} \cap F_{2}^{\perp} \cap F_{3}^{\perp} M_{2}=F_{1}^{\perp} \cap F_{2} \cap F_{3}^{\perp}, M_{3}=F_{1}^{\perp} \cap F_{2}^{\perp} \cap F_{3}$ and $L=F_{1}^{\perp} \cap F_{2}^{\perp} \cap F_{3}^{\perp}$. But we should be careful that the isomorphism by an invertible operator does not preserve the orthogonality.

The aim of our paper is to extend the Brenner's theorem to a certain class of three subspaces in an infinite-dimensional Hilbert space.

The above Brenner's theorem says that any system of three subspaces of a finite-dimensional Hilbert space is decomposed as a direct sum of a distributive part (or Boolean part)

$$
S \oplus N_{1} \oplus N_{2} \oplus N_{3} \oplus M_{1} \oplus M_{2} \oplus M_{3} \oplus L
$$


and a non-distributive part $Q$. Furthermore the non-distributive part $Q=K \oplus K$ has a typical form

$$
(K \oplus K ; K \oplus 0,0 \oplus K,\{(x, x) \mid x \in K\})
$$

of double triangle. The double triangle is the only obstruction of distributive law in finite-dimensional case. We study this type of decomposition for a certain class of systems of three subspaces for an infinite-dimensional Hilbert space. In order to proceed this type of decomposition, we should recall the following basic facts on the subspace lattice structure: In general, a lattice is distributive if and only if it has neither a double triangle nor a pentagon as a sublattice, see [11 for example. In the subspace lattices of an infinite dimensional Hilbert space, there occur both double triangles and pentagons. A von Neumann algebra $M$ is commutative if and only if the lattice of the projections in $M$ is distributive. A von Neumann algebra $M$ is finite if and only if the lattice of the projections in $M$ has no pentagons if and only if the lattice of the projections in $M$ is modular. Therefore we understand that the general case is far beyond having a Brenner type decomposition.

For any bounded linear operator $A$ on a Hilbert space $K$, we can associate a system $\mathcal{S}_{A}$ of four subspaces in $H=K \oplus K$ by

$$
\mathcal{S}_{A}=(H ; K \oplus 0,0 \oplus K, \operatorname{graph} A,\{(x, x) ; x \in K\}) .
$$

Two such systems $\mathcal{S}_{A}$ and $\mathcal{S}_{B}$ are isomorphic if and only if the two operators $A$ and $B$ are similar. The direct sum of such systems corresponds to the direct sum of the operators. In this sense the theory of operators is included into the theory of relative positions of four subspaces. In particular on a finite dimensional space, Jordan blocks correspond to indecomposable systems. Moreover on an infinite dimensional Hilbert space, the above system $\mathcal{S}_{A}$ is indecomposable if and only if $A$ is strongly irreducible, which is an infinite-dimensional analog of a Jordan block, see, for example, a monograph by Jiang and Wang [19].

Halmos initiated the study of transitive lattices and gave an example of transitive lattice consisting of seven subspaces in [16]. HarrisonRadjavi-Rosenthal [17] constructed a transitive lattice consisting of six subspaces using the graph of an unbounded closed operator. HadwinLongstaff-Rosenthal found a transitive lattice of five non-closed linear subspaces in [14]. Any finite transitive lattice which consists of $n$ subspaces of a Hilbert space $H$ gives an indecomposable system of $n-2$ subspaces by withdrawing 0 and $H$, but the converse is not true. It is still unknown whether or not there exists a transitive lattice consisting of five subspaces. Therefore it is also an interesting problem to know whether there exists an indecomposable system of three subspaces in an infinite-dimensional Hilbert space.

Throughout the paper a projection means an operator $e$ with $e^{2}=$ $e=e^{*}$ and an idempotent means an operator $p$ with $p^{2}=p$. The 
direct $\operatorname{sum} \oplus$ is the orthogonal direct sum and $\oplus_{\text {alg }}$ is the algebraic direct sum. The subspace mostly means closed subspace except the algebraic direct sum.

There seems to be interesting relations with the study of representations of $*$-algebras generated by idempotents by S. Kruglyak and Y. Samoilenko [22] and the study on sums of projections by S. Kruglyak, V. Rabanovich and Y. Samoilenko [21]. But we do not know the exact implication, because their objects are different with ours.

In finite dimensional case, the classification of four subspaces is described as the classification of the representations of the extended Dynkin diagram $D_{4}^{(1)}$. Recall that Gabriel [9] listed Dynkin diagrams $A_{n}, D_{n}, E_{6}, E_{7}, E_{8}$ in his theory on finiteness of indecomposable representations of quivers. We discussed on indecomposable representations of quivers on infinite-dimensional Hilbert spaces [7]. We are also under the influence of subfactor theory by Jones [20].

Our study also has a relation with $C^{*}$-algebras generated by idempotents or projections. See Bottcher, Gohberg, Karlovich, Krupnik, Roch, Silbermann and Spittovsky [2] , Hu and Xue [18] and references there.

After we completed our paper, we noticed that there might be a connection with Kadison-Singer algebras introduced by Ge and Yuan [12] and [13].

The authors are supported by JSPS KAKENHI Grant number 23654053 and 25287019 .

\section{SYSTEMS OF $n$ SUBSPACES}

We introduce some basic definitions and facts on the relative position of $n$ subspaces in a separable Hilbert space. Let $H$ be a Hilbert space and $E_{1}, \ldots E_{n}$ be $n$ subspaces in $H$. Then we say that $\mathcal{S}=$ $\left(H ; E_{1}, \ldots, E_{n}\right)$ is a system of $n$-subspaces in $H$ or an $n$-subspace system in $H$. Let $\mathcal{T}=\left(K ; F_{1}, \ldots, F_{n}\right)$ be another system of $n$-subspaces in a Hilbert space $K$. Then $\varphi: \mathcal{S} \rightarrow \mathcal{T}$ is called a homomorphism if $\varphi: H \rightarrow K$ is a bounded linear operator satisfying that $\varphi\left(E_{i}\right) \subset F_{i}$ for $i=1, \ldots, n$. And $\varphi: \mathcal{S} \rightarrow \mathcal{T}$ is called an isomorphism if $\varphi: H \rightarrow K$ is an invertible (i.e., bounded bijective) linear operator satisfying that $\varphi\left(E_{i}\right)=F_{i}$ for $i=1, \ldots, n$. We say that systems $\mathcal{S}$ and $\mathcal{T}$ are isomorphic if there is an isomorphism $\varphi: \mathcal{S} \rightarrow \mathcal{T}$. This means that the relative positions of $n$ subspaces $\left(E_{1}, \ldots, E_{n}\right)$ in $H$ and $\left(F_{1}, \ldots, F_{n}\right)$ in $K$ are same under disregarding angles. We say that systems $\mathcal{S}$ and $\mathcal{T}$ are $u n i$ tarily equivalent if the above isomorphism $\varphi: H \rightarrow K$ can be chosen to be a unitary. This means that the relative positions of $n$ subspaces $\left(E_{1}, \ldots, E_{n}\right)$ in $H$ and $\left(F_{1}, \ldots, F_{n}\right)$ in $K$ are same with preserving the angles between the subspaces. We are interested in the relative position of subspaces up to isomorphism to study the still-remaining fundamental feature of the relative position after disregarding "the angles" . 
We denote by $\operatorname{Hom}(\mathcal{S}, \mathcal{T})$ the set of homomorphisms of $\mathcal{S}$ to $\mathcal{T}$ and $\operatorname{End}(\mathcal{S}):=\operatorname{Hom}(\mathcal{S}, \mathcal{S})$ the set of endomorphisms on $\mathcal{S}$.

Let $G_{2}=\mathbb{Z} / 2 \mathbb{Z} * \mathbb{Z} / 2 \mathbb{Z}=\left\langle a_{1}, a_{2}\right\rangle$ be the free product of the cyclic groups of order two with generators $a_{1}$ and $a_{2}$. For two subspaces $E_{1}$ and $E_{2}$ of a Hilbert space $H$, let $e_{1}$ and $e_{2}$ be the projections onto $E_{1}$ and $E_{2}$. Then $u_{1}=2 e_{1}-I$ and $u_{2}=2 e_{2}-I$ are self-adjoint unitaries. Thus there is a bijective correspondence between the set $\mathcal{S} y s^{2}(H)$ of systems $\mathcal{S}=\left(H ; E_{1}, E_{2}\right)$ of two subspaces in a Hilbert space $H$ and the set $\operatorname{Rep}\left(G_{2}, H\right)$ of unitary representations $\pi$ of $G_{2}$ on $H$ such that $\pi\left(a_{1}\right)=u_{1}$ and $\pi\left(a_{2}\right)=u_{2}$. Similarly let $G_{n}=\mathbb{Z} / 2 \mathbb{Z} * \ldots * \mathbb{Z} / 2 \mathbb{Z}$ be the $n$-times free product of the cyclic groups of order two. Then there is a bijective correspondence between the set $\mathcal{S} y s^{n}(H)$ of systems of $n$ subspaces in a Hilbert space $H$ and the set $\operatorname{Rep}\left(G_{n}, H\right)$ of unitary representations of $G_{n}$ on $H$.

Example 1. Let $H=\mathbb{C}^{2}$. Fix an angle $\theta$ with $0<\theta<\pi / 2$. Put $E_{1}=\{\lambda(1,0) \mid \lambda \in \mathbb{C}\}$ and $E_{2}=\{\lambda(\cos \theta, \sin \theta) \mid \lambda \in \mathbb{C}\}$. Then $\mathcal{S}_{1}=\left(H ; E_{1}, E_{2}\right)$ is isomorphic to $\mathcal{S}_{2}=\left(\mathbb{C}^{2} ; \mathbb{C} \oplus 0,0 \oplus \mathbb{C}\right)$. But the corresponding two unitary representations $\pi_{1}$ and $\pi_{2}$ are not similar, because $\frac{1}{2}\left(\pi_{1}\left(a_{1}\right)+1\right) \frac{1}{2}\left(\pi_{1}\left(a_{2}\right)+1\right) \neq 0$ and $\frac{1}{2}\left(\pi_{2}\left(a_{1}\right)+1\right) \frac{1}{2}\left(\pi_{2}\left(a_{2}\right)+1\right)=$ 0 .

We start with known facts to recall some notation. See [6] for example.

Let $H$ be a Hilbert space and $H_{1}$ and $H_{2}$ be two subspaces of $H$. We write $H_{1} \vee H_{2}:=\overline{H_{1}+H_{2}}$ and $H_{1} \wedge H_{2}:=H_{1} \cap H_{2}$. Then the set of (closed) subspaces of $H$ forms a lattice under these operations $\vee$ and $\wedge$. Two subspaces $H_{1}$ and $H_{2}$ are said to be topologically complementary if $H=H_{1} \vee H_{2}$ and $H_{1} \wedge H_{2}=0$. Two subspaces $H_{1}$ and $H_{2}$ are said to be algebraically complementary if $H=H_{1}+H_{2}$ and $H_{1} \cap H_{2}=0$.

Lemma 2.1. Let $H$ be a Hilbert space and $H_{1}$ and $H_{2}$ be two subspaces of $H$. Then the following are equivalent:

(1) $H_{1}$ and $H_{2}$ are algebraically complementary, i.e., $H=H_{1}+H_{2}$ and $H_{1} \cap H_{2}=0$.

(2) There exists a closed subspace $M \subset H$ such that $\left(H ; H_{1}, H_{2}\right)$ is isomorphic to $\left(H ; M, M^{\perp}\right)$

(3) There exists an idempotent $P \in B(H)$ such that $H_{1}=\operatorname{Im} P$ and $H_{2}=\operatorname{Im}(1-P)$.

Lemma $2.2([6])$. Let $H$ and $K$ be Hilbert spaces and $E \subset H$ and $F \subset K$ be closed subspaces of $H$ and $K$. Let $e \in B(H)$ and $f \in B(K)$ be the projections onto $E$ and $F$. Then the following are equivalent:

(1) There exists an invertible operator $T: H \rightarrow K$ such that $T(E)=F$.

(2) There exists an invertible operator $T: H \rightarrow K$ such that $e=$ $\left(T^{-1} f T\right) e$ and $f=\left(T e T^{-1}\right) f$. 
Using the above lemma, we can describe an isomorphism between two systems of $n$ subspaces in terms of operators only as follows:

Corollary 2.3. Let $\mathcal{S}=\left(H ; E_{1}, \cdots, E_{n}\right)$ and $\mathcal{S}^{\prime}=\left(H^{\prime} ; E_{1}^{\prime}, \cdots, E_{n}^{\prime}\right)$ be two systems of n-subspaces. Let $e_{i}$ (resp. $\left.e_{i}^{\prime}\right)$ be the projection onto $E_{i}$ (resp. $\left.E_{i}^{\prime}\right)$. Then two systems $\mathcal{S}$ and $\mathcal{S}^{\prime}$ are isomorphic if and only if there exists an invertible operator $T: H \rightarrow H^{\prime}$ such that $e_{i}=$ $\left(T^{-1} e_{i}^{\prime} T\right) e_{i}$ and $e_{i}^{\prime}=\left(T e_{i} T^{-1}\right) e_{i}^{\prime}$ for $i=1, \ldots, n$.

Remark. If there exists an invertible operator $T: H \rightarrow H^{\prime}$ such that $e_{i}^{\prime}=T e_{i} T^{-1}$ for $i=1, \ldots, n$, then two systems $\mathcal{S}$ and $\mathcal{S}^{\prime}$ are isomorphic. But the converse is not true as in Example 1.

\section{INDECOMPOSABLE SYSTEMS}

In this section we shall introduce a notion of indecomposable system, that is, a system which cannot be decomposed into a direct sum of smaller systems anymore.

Definition (direct sum). Let $\mathcal{S}=\left(H ; E_{1}, \ldots, E_{n}\right)$ and $\mathcal{S}^{\prime}=\left(H^{\prime} ; E_{1}^{\prime}, \cdots, E_{n}^{\prime}\right)$ be systems of $n$ subspaces in Hilbert spaces $H$ and $H^{\prime}$. Then their direct sum $\mathcal{S} \oplus \mathcal{S}^{\prime}$ is defined by

$$
\mathcal{S} \oplus \mathcal{S}^{\prime}:=\left(H \oplus H^{\prime} ; E_{1} \oplus E_{1}^{\prime}, \ldots, E_{n} \oplus E_{n}^{\prime}\right) .
$$

Definition. (indecomposable system). A system $\mathcal{S}=\left(H ; E_{1}, \ldots, E_{n}\right)$ of $n$ subspaces is called decomposable if the system $\mathcal{S}$ is isomorphic to a direct sum of two non-zero systems. A system $\mathcal{S}=\left(H ; E_{1}, \cdots, E_{n}\right)$ is said to be indecomposable if it is not decomposable.

Example 2. Let $H=\mathbb{C}^{2}$. Fix an angle $\theta$ with $0<\theta<\pi / 2$. Put $E_{1}=\{\lambda(1,0) \mid \lambda \in \mathbb{C}\}$ and $E_{2}=\{\lambda(\cos \theta, \sin \theta) \mid \lambda \in \mathbb{C}\}$. Then $\left(H ; E_{1}, E_{2}\right)$ is isomorphic to

$$
\left(\mathbb{C}^{2} ; \mathbb{C} \oplus 0,0 \oplus \mathbb{C}\right) \cong(\mathbb{C} ; \mathbb{C}, 0) \oplus(\mathbb{C} ; 0, \mathbb{C}) .
$$

Hence $\left(H ; E_{1}, E_{2}\right)$ is decomposable.

Remark. Let $e_{1}$ and $e_{2}$ be the projections onto $E_{1}$ and $E_{2}$ in the Example above. Then the $C^{*}$-algebra $C^{*}\left(\left\{e_{1}, e_{2}\right\}\right)$ generated by $e_{1}$ and $e_{2}$ is exactly $B(H) \cong M_{2}(\mathbb{C})$. Therefore the irreducibility of $C^{*}\left(\left\{e_{1}, e_{2}\right\}\right)$ does not imply the indecomposability of $\left(H ; E_{1}, E_{2}\right)$. Thus seeking an indecomposable system of subspaces is much more difficult and fundamental task than showing irreducibility of the $C^{*}$-algebra generated by the corresponding projections for the subspaces.

We can characterize decomposability of systems inside the ambient Hilbert space as in [6]

Let $H$ be a Hilbert space and $\mathcal{S}=\left(H ; E_{1}, \ldots, E_{n}\right)$ a system of $n$ subspaces. Then the following conditions are equivalent: 
(1) $\mathcal{S}$ is decomposable.

(2) there exist non-zero closed subspaces $H_{1}$ and $H_{2}$ of $H$ such that $H_{1}+H_{2}=H, H_{1} \cap H_{2}=0$ and $E_{i}=E_{i} \cap H_{1}+E_{i} \cap H_{2}$ for any $i=1, \ldots, n$.

We give a condition of decomposability in terms of endomorphism algebras for the systems.

We put $\operatorname{Idem}(\mathcal{S}):=\left\{T \in \operatorname{End}(\mathcal{S}) ; T=T^{2}\right\}$.

Let $\mathcal{S}=\left(H ; E_{1}, \ldots, E_{n}\right)$ be a system of $n$ subspaces in a Hilbert space $H$. Then $\mathcal{S}$ is indecomposable if and only if $\operatorname{Idem}(\mathcal{S})=\{0, I\}$.

Let $\mathcal{S}=\left(H ; E_{1}, \ldots, E_{n}\right)$ be a system of $n$ subspaces in a Hilbert space $H$. Let $e_{i}$ be the projection of $H$ onto $E_{i}$ for $i=1, \ldots, n$. If $\mathcal{S}=\left(H ; E_{1}, \ldots, E_{n}\right)$ is indecomposable, then the $C^{*}\left(\left\{e_{1}, \ldots, e_{n}\right\}\right)$ generated by $e_{1}, \ldots, e_{n}$ is irreducible. But the converse is not true.

Definition. Let $\mathcal{S}=\left(H ; E_{1}, \ldots, E_{n}\right)$ be a system of $n$ subspaces in a Hilbert space $H$. Let $e_{i}$ be the projection of $H$ onto $E_{i}$ for $i=$ $1, \ldots, n$. We say that $\mathcal{S}$ is a commutative system if the $C^{*}\left(\left\{e_{1}, \ldots, e_{n}\right\}\right)$ generated by $e_{1}, \ldots, e_{n}$ is commutative. Be careful that commutativity is not an isomorphic invariant as shown in Example 1. But it makes sense that a system is isomorphic to a commutative system.

Let $\mathcal{S}=\left(H ; E_{1}, \ldots, E_{n}\right)$ be a system of $n$ subspaces in a Hilbert space $H$. Assume that $\mathcal{S}$ is a commutative system. Then $\mathcal{S}$ is indecomposable if and only if $\operatorname{dim} H=1$. Moreover each subset $\Lambda \subset\{1, \ldots, n\}$ corresponds to a commutative system satisfying $\operatorname{dim} E_{i}=1$ for $i \in \Lambda$ and $\operatorname{dim} E_{i}=0$ for $i \notin \Lambda$.

Example 3. Let $H=\mathbb{C}^{2}$. Put $E_{1}=\mathbb{C} \oplus 0, E_{2}=0 \oplus \mathbb{C}$ and $E_{3}=\{(x, x) x \in \mathbb{C}\}$. Then $\mathcal{S}=\left(H ; E_{1}, E_{2}, E_{3}\right)$ is indecomposable. The system $\mathcal{S}$ is the lowest dimensional one among non-commutative indecomposable systems. In fact, the system $\mathcal{S}$ forms a double triangle in the sense below. We see that distributive law fails:

$$
\left(E_{1} \vee E_{2}\right) \wedge E_{3} \neq\left(E_{1} \wedge E_{2}\right) \vee\left(E_{1} \wedge E_{3}\right)
$$

Definition. We say that a system $\mathcal{S}=\left(H ; E_{1}, E_{2}, E_{3}\right)$ of three subspaces in a Hilbert space $H$ forms a double triangle if the family $\left\{H, E_{1}, E_{2}, E_{3}, 0\right\}$ is a double triangle lattice, (which is also called a diamond), that is,

$$
E_{i} \vee E_{j}=H, \quad \text { and } \quad E_{i} \wedge E_{j}=0, \quad(i \neq j, i, j=1,2,3) .
$$

and each $E_{i} \neq H, E_{i} \neq 0$. We remark that the distributive law fails in any double triangle.

$$
\left(E_{1} \vee E_{2}\right) \wedge E_{3} \neq\left(E_{1} \wedge E_{2}\right) \vee\left(E_{1} \wedge E_{3}\right)
$$

Example 4. Let $G=\mathbb{Z} / 2 \mathbb{Z} * \mathbb{Z} / 2 \mathbb{Z} * \mathbb{Z} / 2 \mathbb{Z}=\left\langle a_{1}, a_{2}, a_{3}\right\rangle$ be the free product of the cyclic groups of order two with three generators 
$a_{1}, a_{2}$ and $a_{3}$. Let $\lambda$ be the left regular representation of $G$ on $H=$ $\ell^{2}(G)$. Then the reduced group $C^{*}$-algebra $C_{r}^{*}(G)$ is generatedby $\lambda_{a_{1}}$, $\lambda_{a_{2}}$ and $\lambda_{a_{3}}$. Since these three generators are self-adjoint unitaries, $e_{i}:=\left(\lambda_{a_{i}}+I\right) / 2,(i=1,2,3)$ are projections. Let $E_{i}=\operatorname{Im} e_{i}$. Then a system $\mathcal{S}=\left(H ; E_{1}, E_{2}, E_{3}\right)$ of three subspaces forms a double triangle. In fact, let $x=\sum_{g} x_{g} \delta_{g} \in E_{1} \cap E_{2}$. Since $e_{i} x=x$, we have $\lambda_{a_{i}} x=x$ for $i=1,2$. Therefore $x_{a_{i} g}=x_{g}$ for any $g \in G$. Since $\sum_{h}\left|x_{h}\right|^{2}<\infty$, $x_{g}=0$ for any $g$. Therefore $x=0$. Hence $E_{1} \cap E_{2}=O$. The other conditions are similarly checked.

Definition. We say that a system $\mathcal{S}=\left(H ; E_{1}, E_{2}, E_{3}\right)$ of three subspaces in a Hilbert space $H$ forms a pentagon (with $E_{3} \supset E_{2}$ ) if the family $\left\{H, E_{1}, E_{2}, E_{3}, 0\right\}$ is a pentagon lattice (with $E_{3} \supset E_{2}$ ), that is,

$$
E_{1} \vee E_{2}=H, \quad E_{1} \wedge E_{3}=0, \text { and } E_{3} \supset E_{2} \text { with } E_{3} \neq E_{2},
$$

and each $E_{i} \neq H, E_{i} \neq 0$. We also say that $\mathcal{S}=\left(H ; E_{1}, E_{2}, E_{3}\right)$ is a pentagon system.

Example 5. Let $K$ be a Hilbert space and $A: K \rightarrow K$ a bounded operator such that $A$ is one to one and $\operatorname{Im} A$ is dense in $K$ and not equal to $K$. Put $H=K \oplus K, E_{1}=K \oplus 0$ and $E_{2}=\{(x, A x) \mid x \in K\}$. Let $M \neq 0$ be a finite-dimensional subspace of $K$ such that $M \cap \operatorname{Im} A=0$. Put $E_{3}=E_{2}+(O, M)$. Then $\mathcal{S}=\left(H ; E_{1}, E_{2}, E_{3}\right)$ is a pentagon system.

Recall that Halmos initiated the study of transitive lattices. A complete lattice of closed subspaces of a Hilbert space $H$ containing 0 and $H$ is called transitive if every bounded operator on $H$ leaving each subspace invariant is a scalar multiple of the identity. Halmos gave an example of transitive lattice consisting of seven subspaces in [16]. Harrison-Radjavi-Rosenthal [17] constructed a transitive lattice consisting of six subspaces using the graph of an unbounded operator. Any finite transitive lattice which consists of $n$ subspaces gives an indecomposable system of $n$ - 2 subspaces but the converse is not true. Following the study of transitive lattices, we shall introduce the notion of transitive system.

Definition. Let $\mathcal{S}=\left(H ; E_{1}, \ldots, E_{n}\right)$ be a system of $n$ subspaces in a Hilbert space $H$. Then we say that $\mathcal{S}$ is transitive if $\operatorname{End}(\mathcal{S})=\mathbb{C} I_{H}$. Recall that $\mathcal{S}$ is indecomposable if and only if $\operatorname{Idem}(\mathcal{S})=\{0, I\}$. Hence if $\mathcal{S}$ is transitive, then $\mathcal{S}$ is indecomposable. But the converse is not true. In fact, the system

$$
\mathcal{S}_{S}=(H ; K \oplus 0,0 \oplus K, \operatorname{graph} A,\{(x, x) ; x \in K\}) .
$$

of four subspaces associated with a unilateral shift $S$ as above is indecomposable but is not transitive, because $\operatorname{End}(\mathcal{S})$ contains $S \oplus S$.

Example 6. (Harrison-Radjavi-Rosenthal [17]) Let $K=\ell^{2}(\mathbb{Z})$ and $H=$ $K \oplus K$. Consider a sequence $\left(\alpha_{n}\right)_{n}$ given by $\alpha_{n}=1$ for $n \leq 0$ and 
$\alpha_{n}=\exp \left((-1)^{n} n !\right)$ for $n \geq 1$. Consider a bilateral weighted shift $S: \mathcal{D}_{T} \rightarrow K$ such that $T\left(x_{n}\right)_{n}=\left(\alpha_{n-1} x_{n-1}\right)_{n}$ with the domain $\mathcal{D}_{T}=$ $\left\{\left(x_{n}\right)_{n} \in \ell^{2}(\mathbb{Z}) ; \sum_{n}\left|\alpha_{n} x_{n}\right|^{2}<\infty\right\}$. Let $E_{1}=K \oplus 0, E_{2}=0 \oplus K$, $E_{3}=\left\{(x, T x) \in H ; x \in \mathcal{D}_{T}\right\}$ and $E_{4}=\{(x, x) \in H ; x \in K\}$. Harrison, Radjavi and Rosenthal showed that $\left\{0, H, E_{1}, E_{2}, E_{3}, E_{4}\right\}$ is a transitive lattice. Hence the system $\mathcal{S}=\left(H ; E_{1}, E_{2}, E_{3}, E_{4}\right)$ of four subspaces in $H$ is transitive and in particular indecomposable.

It is easy to see the case of indecomposable systems of one subspace even in an infinite-dimensional Hilbert space.

Let $H$ be a Hilbert space and $\mathcal{S}=(H ; E)$ a system of one subspace. Then $\mathcal{S}=(H ; E)$ is indecomposable if and only if $\mathcal{S} \cong(\mathbb{C} ; 0)$ or $\mathcal{S} \cong$ $(\mathbb{C} ; \mathbb{C})$.

Let $\mathcal{S}=(H ; E)$ and $\mathcal{S}^{\prime}=\left(H^{\prime} ; E^{\prime}\right)$ be two systems of one subspace. Then $\mathcal{S}$ and $\mathcal{S}^{\prime}$ are isomorphic if and only if $\operatorname{dim} E=\operatorname{dim} E^{\prime}$ and $\operatorname{codim} E=\operatorname{codim} E^{\prime}$.

It is a well known fact that the relative position of two subspaces $E_{1}$ and $E_{2}$ in a Hilbert space $H$ can be described completely up to unitary equivalence. The Hilbert space $H$ is the direct sum of five subspaces:

$$
H=\left(E_{1} \cap E_{2}\right) \oplus \text { (the rest) } \oplus\left(E_{1} \cap E_{2}^{\perp}\right) \oplus\left(E_{1}^{\perp} \cap E_{2}\right) \oplus\left(E_{1}^{\perp} \cap E_{2}^{\perp}\right) .
$$

In the rest part, $E_{1}$ and $E_{2}$ are in generic position and the relative position is described only by "the angles" between them. In fact the rest part is written as $K \oplus K$ for some subspace $K$ and there exist two positive operators $c, s \in B(K)$ with null kernels with $c^{2}+s^{2}=1$ such that

$$
E_{1}=\left(E_{1} \cap E_{2}\right) \oplus \operatorname{Im}\left(\begin{array}{ll}
1 & 0 \\
0 & 0
\end{array}\right) \oplus\left(E_{1} \cap E_{2}^{\perp}\right) \oplus 0 \oplus 0,
$$

and

$$
E_{2}=\left(E_{1} \cap E_{2}\right) \oplus \operatorname{Im}\left(\begin{array}{cc}
c^{2} & c s \\
c s & s^{2}
\end{array}\right) \oplus 0 \oplus\left(E_{1}^{\perp} \cap E_{2}\right) \oplus 0 .
$$

By the functional calculus, there exists a unique positive operator $\theta$, called the angle operator, such that $c=\cos \theta$ and $s=\sin \theta$ with $0 \leq \theta \leq \frac{\pi}{2}$. We see that the algebraic sum $E_{1}+E_{2}$ is closed if and only if $s c K+s^{2} K=K$ if and only if $s$ is invertible. And $E_{1}+E_{2}^{\perp}$ is closed if and only if $E_{1}^{\perp}+E_{2}$ is closed if and only if $c$ is invertible. We need the following fact:

Lemma 3.1. Let $E_{1}$ and $E_{2}$ be two subspaces in a Hilbert space $H$. Let $P_{i}$ be the projection of $H$ onto $E_{i}$. If $E_{1}+E_{2}$ is closed, then $T:=\left.\left(P_{1}+P_{2}\right)\right|_{\left(E_{1}+E_{2}\right)}: E_{1}+E_{2} \rightarrow E_{1}+E_{2}$ is an onto invertible operator.

Proof. Since $E_{1}+E_{2}$ is closed, $s$ is invertible. Then it is easy to see that

$$
T=I \oplus\left(\begin{array}{cc}
I+c^{2} & c s \\
c s & s^{2}
\end{array}\right) \oplus I \oplus I
$$


is invertible, because the non-trivial component has the operator determinant $\left(I+c^{2}\right) s^{2}-c s c s=s^{2}$.

Let $\mathcal{S}=\left(H ; E_{1}, E_{2}\right)$ be a system of two subspaces in a Hilbert space $H$. Then $\mathcal{S}$ is indecomposable if and only if $\mathcal{S}$ is isomorphic to one of the following four commutative systems:

$$
\mathcal{S}_{1}=(\mathbb{C} ; \mathbb{C}, 0), \quad \mathcal{S}_{2}=(\mathbb{C} ; 0, \mathbb{C}), \mathcal{S}_{3}=(\mathbb{C} ; \mathbb{C}, \mathbb{C}), \quad \mathcal{S}_{4}=(\mathbb{C} ; 0,0)
$$

\section{Brenner type DeCOMPOSITION}

We introduce a Brenner type decomposition which is a generalization of a Brenner decomposition of a system of three subspaces in a finite dimensional Hilbert space.

Definition. Let $\mathcal{S}=\left(H ; E_{1}, E_{2}, E_{3}\right)$ be a system of three subspaces in a Hilbert space $H$. Then $\mathcal{S}$ is said to have a Brenner type decomposition if $\mathcal{S}$ is isomorphic to a system $\mathcal{T}=\left(H ; F_{1}, F_{2}, F_{3}\right)$ satisfying that there exist subspaces $S, N_{1}, N_{2}, N_{3}, M_{1}, M_{2}, M_{3}, Q, L$ of $H$ such that $\left(Q ; Q_{1}, Q_{2}, Q_{3}\right)$ forms a double triangle and

$$
\begin{aligned}
& H=S \oplus N_{1} \oplus N_{2} \oplus N_{3} \oplus M_{1} \oplus M_{2} \oplus M_{3} \oplus Q \oplus L \\
& F_{1}=S \oplus 0 \quad \oplus N_{2} \oplus N_{3} \oplus M_{1} \oplus 0 \quad \oplus 0 \quad \oplus Q_{1} \oplus 0 \\
& F_{2}=S \oplus N_{1} \oplus 0 \quad \oplus N_{3} \oplus 0 \quad \oplus M_{2} \oplus 0 \quad \oplus Q_{2} \oplus 0 \\
& F_{3}=S \oplus N_{1} \oplus N_{2} \oplus 0 \quad \oplus 0 \quad \oplus 0 \quad \oplus M_{3} \oplus Q_{3} \oplus 0
\end{aligned}
$$

Proposition 4.1. Let $\mathcal{S}=\left(H ; E_{1}, E_{2}, E_{3}\right)$ be a system of three subspaces in a Hilbert space $H$. Assume that $\left(H ; E_{1}, E_{2}, E_{3}\right)$ forms a double triangle. Then the followings are equivalent:

(1) Linear sums $E_{i}+E_{j}$ are closed for any $i, j \in\{1,2,3\}$ with $i \neq j$.

(2) $\mathcal{S}$ is isomorphic to a typical form, i.e.

$$
\left(H ; H_{1}, H_{2}, H_{3}\right) \cong(K \oplus K ; K \oplus 0,0 \oplus K,\{(x, x) \mid x \in K\})
$$

for some Hilbert space $K$.

Proof. It is trivial that (2) implies (1). Conversely, assume (1). Since $H=E_{1}+E_{2}$ and $E_{1} \cap E_{2}=0$, we may and do assume that $E_{2}=E_{1}^{\perp}$ up to isomorphism. Apply two subspace theorem for $E_{1}$ and $E_{3}$. Since $E_{1} \cap E_{3}=0$ and $E_{1}^{\perp} \cap E_{3}^{\perp}=0$, The Hilbert space $H$ is the direct sum of three subspaces:

$$
H=(K \oplus K) \oplus\left(E_{1} \cap E_{3}^{\perp}\right) \oplus\left(E_{1}^{\perp} \cap E_{3}\right) .
$$

for some subspace $K$ and there exist two positive operators $c, s \in B(K)$ with null kernels with $c^{2}+s^{2}=1$ such that

$$
E_{1}=\operatorname{Im}\left(\begin{array}{ll}
1 & 0 \\
0 & 0
\end{array}\right)_{10} \oplus\left(E_{1} \cap E_{3}^{\perp}\right) \oplus 0,
$$


and

$$
E_{3}=\operatorname{Im}\left(\begin{array}{cc}
c^{2} & c s \\
c s & s^{2}
\end{array}\right) \oplus 0 \oplus\left(E_{1}^{\perp} \cap E_{3}\right) .
$$

Since $E_{2} \cap E_{3}=0$, we have that $E_{1}^{\perp} \cap E_{3}=0$. Since $E_{2} \vee E_{3}=H$, we have that $E_{1} \cap E_{3}^{\perp}=0$. Moreover

$$
\operatorname{Im}\left(\begin{array}{cc}
c^{2} & c s \\
c s & s^{2}
\end{array}\right)=\left\{\left(\begin{array}{l}
c z \\
s z
\end{array}\right) \mid z \in K\right\}
$$

because $c+s$ is invertible.

Since $E_{1}+E_{3}$ is closed, $s$ is invertible. Since $E_{1}^{\perp}+E_{3}=E_{2}+E_{3}$ is closed, $c$ is invertible. Consider an invertible operator

$$
T=\left(\begin{array}{cc}
c^{-1} & 0 \\
0 & s^{-1}
\end{array}\right)
$$

Then $T E_{1}=K \oplus 0, T E_{2}=0 \oplus K$ and $T E_{3}=\left\{\left(\begin{array}{l}z \\ z\end{array}\right) \mid z \in K\right\}$. This completes the proof.

We need the following Theorem after [ 8 , Corollary 4.1] by Feshchenko who studies closedness of the sum of $n$ subspaces of a Hilbert space. Let $H_{1}, \ldots, H_{n}$ be subspaces of a Hilbert space. Then $H_{1}, \ldots, H_{n}$ are said to be linearly independent if for any $x_{i} \in H_{i}(i=1, \ldots, n)$, if $x_{1}+\ldots x_{n}=0$, then $x_{1}=\cdots=x_{n}=0$. They are linearly independent if and only if the representation $x=x_{1}+\cdots+x_{n}$ for $x_{i} \in H_{i}$ $(i=1, \ldots, n)$ is unique if and only if

$$
H_{i} \cap\left(\sum_{\{j ; j \neq i\}} H_{j}\right)=0
$$

for any $i=1, \ldots, n$.

Theorem $4.2([8])$. Let $H_{1}, \ldots, H_{n}$ be linear independent subspaces of a Hilbert space $H$. If $H=H_{1}+\cdots+H_{n}$, then for any collection of subscripts $i(1), \ldots, i(k)$, the sum $H_{i(1)}+\cdots+H_{i(k)}$ is closed.

Using the Feshchenko's Theorem above, we can extend Lemma 2.1 to $n$-subspaces.

Theorem 4.3. Let $H_{1}, \ldots, H_{n}$ be $n$-subspaces of a Hilbert space $H$. Then the following are equivalent:

(1) $H=H_{1}+\cdots+H_{n}$ and $H_{1}, \ldots, H_{n}$ are linearly independent.

(2) $H$ is isomorphic to an outer orthogonal sum $H_{1} \oplus H_{2} \cdots \oplus H_{n}$, i.e., there exists an invertible operator $T: H \rightarrow H_{1} \oplus H_{2} \cdots \oplus H_{n}$ such that $T\left(H_{i}\right)=0 \oplus H_{i} \oplus 0$.

Proof. Assume (1). By the Feshchenko's Theorem above, $H_{1}+H_{2}$ is closed. Since $H_{1}$ and $H_{2}$ are linearly independent, $H_{1}+H_{2}$ is isomorphic to an outer orhtogonal sum $H_{1} \oplus H_{2}$ by Lemma 2.1. Since $\left(H_{1} \oplus H_{2}\right)+H_{3}$ is closed by the Feshchenko's theorem and $\left(H_{1} \oplus H_{2}\right)$ and $H_{3}$ are linearly independent, $\left(H_{1} \oplus H_{2}\right)+H_{3}$ is isomorphic to an outer orhtogonal sum 
$H_{1} \oplus H_{2} \oplus H_{3}$ by Lemma 2.1. Inductively we can show (2). The converse is clear.

The failure of the ditributive law is measured by the inclusions:

$$
\left(\left(E_{i} \wedge E_{j}\right) \vee\left(E_{i} \wedge E_{k}\right)\right) \subset\left(E_{i} \wedge\left(E_{j} \vee E_{k}\right)\right)
$$

Therefore the finite dimensonality of its quotient space is a slight generalization of the finite dimensionality of the ambient space $H$.

Theorem 4.4. Let $\mathcal{S}=\left(H ; E_{1}, E_{2}, E_{3}\right)$ be a system of three subspaces in a Hilbert space $H$. Then the followings are equivalent:

(1) Linear sums $E_{i}+E_{j}$ and $\left(E_{i} \cap E_{k}\right)+\left(E_{j} \cap E_{k}\right)$ are closed for $i, j, k \in\{1,2,3\}$ with $i \neq j \neq k \neq i$ and the quotient space $\left(E_{3} \wedge\left(E_{1} \vee E_{2}\right)\right) /\left(\left(E_{3} \wedge E_{1}\right) \vee\left(E_{3} \wedge E_{2}\right)\right)$ is finite-dimensional.

(2) $\mathcal{S}$ has a Brenner type decomposition with a finite-dimensional double triangle part $Q$.

Moreover if these equivalent conditions are satisfied, then the double triangle part $Q$ is isomorphic to a typical form, i.e.

$$
\left(Q ; Q_{1}, Q_{2}, Q_{3}\right) \cong(K \oplus K ; K \oplus 0,0 \oplus K,\{(x, x) \mid x \in K\})
$$

for some Hilbert space $K$.

Proof. It is trival that (2) implies (1). Conversely, assume (1). Let

$$
Q_{3}=\left(E_{3} \wedge\left(E_{1} \vee E_{2}\right)\right) \cap\left(\left(E_{3} \wedge E_{1}\right) \vee\left(E_{3} \wedge E_{2}\right)\right)^{\perp}
$$

Then $Q_{3}$ is finite-dmensional by the assumption and

$$
\left(E_{3} \wedge\left(E_{1} \vee E_{2}\right)\right)=\left(\left(E_{3} \wedge E_{1}\right) \vee\left(E_{3} \wedge E_{2}\right)\right) \oplus Q_{3}
$$

Let $P_{i}$ be the projection of $H$ onto $E_{i}$. Since $E_{1}+E_{2}$ is closed, $T:=\left.\left(P_{1}+P_{2}\right)\right|_{\left(E_{1}+E_{2}\right)}: E_{1}+E_{2} \rightarrow E_{1}+E_{2}$ is an onto invertible operator by Lemma 3.1. Put $A_{1}=P_{1} T^{-1}$ and $A_{2}=P_{2} T^{-1}$. Then $A_{1}+A_{2}=\left.i d\right|_{E_{1}+E_{2}}$. Put $Q_{1}:=A_{1}\left(Q_{3}\right) \subset E_{1}$ and $Q_{2}:=A_{2}\left(Q_{3}\right) \subset E_{2}$. Then $Q_{1}$ and $Q_{2}$ are finite-dimensional. For any $q_{3} \in Q_{3}$, put $q_{1}=$ $A_{1} q_{3} \in Q_{1}$ and $q_{2}=A_{2} q_{3} \in Q_{2}$. Then $q_{1}+q_{2}=q_{3}$. Let $Q:=Q_{1}+Q_{2}$. Then

$$
Q=Q_{1}+Q_{2}=Q_{2}+Q_{3}=Q_{3}+Q_{1} .
$$

Moreover $Q_{2} \cap Q_{3}=0$. In fact, $Q_{2} \cap Q_{3} \subset E_{2} \cap E_{3}$ and $Q_{2} \cap Q_{3} \subset$ $Q_{3} \subset\left(E_{2} \cap E_{3}\right)^{\perp}$. Similarly we have $Q_{1} \cap Q_{3}=0$. Let $q \in Q_{1} \cap Q_{2}$. Then there exists $q_{3} \in Q_{3}$ such that $q=A_{1} q_{3}$ and

$$
q_{3}=A_{1} q_{3}+A_{2} q_{3}=q+A_{2} q_{3} \in Q_{2}+Q_{2}=Q_{2}
$$

Thus $q_{3} \in Q_{3} \cap Q_{2}=0$. Hence $q=A_{1} q_{3}=0$. This shows that $Q_{1} \cap Q_{2}=0$. Therefore $\left(Q ; Q_{1}, Q_{2}, Q_{3}\right)$ forms a double triangle.

We shall show that

$$
\left(E_{1} \cap\left(E_{2}+E_{3}\right)\right)=\left(\left(E_{1} \cap E_{2}\right)+\left(E_{1} \cap E_{3}\right)\right) \oplus_{\text {alg }} Q_{1}
$$


Since $Q_{1} \subset E_{1}$ and $Q_{1} \subset Q_{2}+Q_{3} \subset\left(E_{2}+E_{3}\right)$,

$$
\left(E_{1} \cap\left(E_{2}+E_{3}\right)\right) \supset\left(\left(E_{1} \cap E_{2}\right)+\left(E_{1} \cap E_{3}\right)\right)+Q_{1}
$$

Conversely let $x_{1} \in\left(E_{1} \cap\left(E_{2}+E_{3}\right)\right)$. Then there exist $x_{2} \in E_{2}$ and $x_{3} \in E_{3}$ such that $x_{1}=x_{2}+x_{3}$. Since

$$
x_{3}=x_{1}-x_{2} \in E_{3} \cap\left(E_{1}+E_{2}\right)=\left(\left(E_{3} \cap E_{1}\right)+\left(E_{3} \cap E_{2}\right)\right)+Q_{3},
$$

there exist $y_{1} \in E_{3} \cap E_{1}, y_{2} \in E_{3} \cap E_{2}$ and $q_{3} \in Q_{3}$ such that $x_{3}=$ $y_{1}+y_{2}+q_{3}$. Since $Q_{3} \subset Q_{1}+Q_{2}$, there exist $q_{1} \in Q_{1}$ and $q_{2} \in Q_{2}$ such that $q_{3}=q_{1}+q_{2}$. Then we have that

$$
x_{1}-x_{2}=x_{3}=y_{1}+y_{2}+q_{3}=y_{1}+y_{2}+q_{1}+q_{2} \text {. }
$$

Put

$$
z_{12}:=x_{1}-y_{1}-q_{1}=y_{2}+x_{2}+q_{2} \in E_{1} \cap E_{2} .
$$

Then

$$
x_{1}=q_{1}+y_{1}+z_{12} \in Q_{1}+E_{3} \cap E_{1}+E_{1} \cap E_{2}
$$

This implies that

$$
\left(E_{1} \cap\left(E_{2}+E_{3}\right)\right) \subset\left(\left(E_{1} \cap E_{2}\right)+\left(E_{1} \cap E_{3}\right)\right)+Q_{1}
$$

We shall show that $\left(\left(E_{1} \cap E_{2}\right)+\left(E_{1} \cap E_{3}\right)\right) \cap Q_{1}=0$. Let $q_{1} \in\left(\left(E_{1} \cap\right.\right.$ $\left.\left.E_{2}\right)+\left(E_{1} \cap E_{3}\right)\right) \cap Q_{1}$. Then there exist $y \in E_{1} \cap E_{2}$ and $z \in E_{1} \cap E_{3}$ such that $q_{1}=y+z$. Since $q_{1} \in Q_{1}$, there exists $q_{3} \in Q_{3}$ such that $q_{1}=A_{1} q_{3}$. Put $q_{2}=A_{2} q_{3}$. Then $q_{3}=q_{1}+q_{2}$. Hence $y+z=q_{1}=q_{3}-q_{2}$. Put

$$
s:=z-q_{3}=-y-q_{2} \in E_{3} \cap E_{2} .
$$

Then $q_{3}=z-s \in\left(E_{3} \cap E_{1}\right)+\left(E_{3} \cap E_{2}\right)$. Hence $q_{3} \in Q_{3} \cap\left(\left(E_{3} \cap E_{1}\right)+\right.$ $\left.\left(E_{3} \cap E_{2}\right)\right)=0$. Thus $q_{1}=A_{1} q_{3}=0$. Therefore we have that

$$
\left(E_{1} \cap\left(E_{2}+E_{3}\right)\right)=\left(\left(E_{1} \cap E_{2}\right)+\left(E_{1} \cap E_{3}\right)\right) \oplus_{\text {alg }} Q_{1}
$$

Similarly we have that

$$
\left(E_{2} \cap\left(E_{1}+E_{3}\right)\right)=\left(\left(E_{2} \cap E_{1}\right)+\left(E_{2} \cap E_{3}\right)\right) \oplus_{\text {alg }} Q_{2}
$$

Put

$$
\begin{aligned}
& M_{1}:=E_{1} \cap\left(E_{1} \cap\left(E_{2}+E_{3}\right)\right)^{\perp} \\
& M_{2}:=E_{2} \cap\left(E_{2} \cap\left(E_{3}+E_{1}\right)\right)^{\perp} \\
& M_{3}:=E_{3} \cap\left(E_{3} \cap\left(E_{1}+E_{2}\right)\right)^{\perp}
\end{aligned}
$$

Then we have that

$$
E_{1}=M_{1} \oplus\left(E_{1} \cap\left(E_{2}+E_{3}\right)\right), \quad E_{2}=M_{2} \oplus\left(E_{2} \cap\left(E_{3}+E_{1}\right)\right)
$$

and

$$
E_{3}=M_{3} \oplus\left(E_{3} \cap\left(E_{1}+E_{2}\right)\right)
$$

Put $S:=E_{1} \cap E_{2} \cap E_{3}$ and

$$
N_{1}:=E_{2} \cap E_{3} \cap S^{\perp}, \quad N_{2}:=E_{3} \cap E_{1} \cap S^{\perp} \quad \text { and } N_{3}:=E_{1} \cap E_{2} \cap S^{\perp} .
$$

Then we have that

$$
E_{2} \cap E_{3}=S \oplus N_{1}, \quad E_{3} \cap E_{1}=\underset{13}{S \oplus N_{2}} \text { and } E_{1} \cap E_{2}=S \oplus N_{3} .
$$


Put $L:=\left(E_{1}+E_{2}+E_{3}\right)^{\perp} \cap H$. Moreover

$$
E_{1}=M_{1}+\left(\left(E_{1} \cap E_{2}\right)+\left(E_{1} \cap E_{3}\right)\right)+Q_{1}=S+N_{2}+N_{3}+M_{1}+Q_{1}
$$

Similarly we also have that

$$
E_{2}=S+N_{1}+N_{3}+M_{2}+Q_{2}, \quad \text { and } E_{3}=S+N_{1}+N_{2}+M_{3}+Q_{3} .
$$

Therefore

$$
E_{1}+E_{2}+E_{3}=S+N_{1}+N_{2}+N_{3}+M_{1}+M_{2}+M_{3}+Q
$$

and

$$
H=\left(E_{1}+E_{2}+E_{3}\right)+L=S+N_{1}+N_{2}+N_{3}+M_{1}+M_{2}+M_{3}+Q+L .
$$

Finally we shall show that the linear sum of the right-hand side is in fact an algebraic direct sum. We need to show that $S, N_{1}, N_{2}, N_{3}, M_{1}, M_{2}, M_{3}, Q$ and $L$ are linearly indepenent. Let

$$
s+n_{1}+n_{2}+n_{3}+m_{1}+m_{2}+m_{3}+q_{1}+q_{2}+\ell=0
$$

for $s \in S, n_{1} \in N_{1}, n_{2} \in N_{2}, n_{3} \in N_{3}, m_{1} \in M_{1}, m_{2} \in M_{2}, m_{3} \in$ $M_{3}, q_{1} \in Q_{1}, q_{2} \in Q_{2}$ and $\ell \in L$. Then it is clear that $\ell=0$. Therefore $-m_{3}=\left(n_{2}+m_{1}+q_{1}\right)+\left(n_{1}+m_{2}+q_{2}\right)+\left(n_{3}+s\right) \in E_{1}+E_{2}+E_{1} \cap E_{2} \subset E_{1}+E_{2}$.

Therefore $m_{3} \in M_{3} \cap\left(E_{3} \cap\left(E_{1}+E_{2}\right)\right)=0$. Thus $m_{3}=0$. Since

$$
q_{1}+q_{2}=q_{2}^{\prime}+q_{3}^{\prime}=q_{3}^{\prime \prime}+q_{1}^{\prime \prime}
$$

for some $q_{2} \in Q_{2}, q_{3}^{\prime}, q_{3}^{\prime \prime} \in Q_{3}$ and $q_{1}^{\prime \prime} \in Q_{1}$, we similarly have that $m_{1}=m_{2}=0$. Hence

$$
s+n_{1}+n_{2}+n_{3}+q_{1}+q_{2}=0 .
$$

Put $w:=n_{1}+q_{2}=-\left(n_{2}+n_{3}+q_{1}\right) \in E_{2} \cap E_{1}$. Then

$$
q_{2}=w-n_{1} \in\left(E_{2} \cap E_{1}\right)+\left(E_{2} \cap E_{3}\right)
$$

Since $q_{2} \in Q_{2} \cap\left(\left(E_{2} \cap E_{1}\right)+\left(E_{2} \cap E_{3}\right)\right)=0$, we have that $q_{2}=0$. Similarly we have that $q_{1}=0$. Therefore $s+n_{1}+n_{2}+n_{3}=0$. Since

$$
E_{1} \cap E_{2} \ni s+n_{3}=-n_{1}-n_{2} \in E_{3},
$$

$s+n_{3} \in\left(E_{1} \cap E_{2}\right) \cap E_{3}=S$. Thus $n_{3} \in S \cap N_{3}=0$. Therefore $n_{3}=0$. Similarly we have that $n_{1}=n_{2}=0$. Hence $s=0$. Finally Theorem 4.3 implies the conclusion. The rest is clear by Proposition 4.1 ,

As a Corollary, we get the original Brenner's theorem.

Corollary 4.5 ([3], 23]). Let $\mathcal{S}=\left(H ; E_{1}, E_{2}, E_{3}\right)$ be a system of three subspaces in a finite dimensional Hilbert space $H$. Then $\mathcal{S}$ has a Brenner type decomposition. 
Remark. Even if an ambient space $H$ is finite-dimensional, a double triangle part is not uniquely determined in a Brenner type decomposition. In fact, let $H=\mathbb{C} \oplus \mathbb{C} \oplus \mathbb{C}, E_{1}=\mathbb{C} \oplus 0 \oplus \mathbb{C}, E_{2}=0 \oplus \mathbb{C} \oplus \mathbb{C}$ and $E_{3}=\{x(1,1,1) \in H \mid x \in \mathbb{C}\}$. Put $N_{3}=0 \oplus 0 \oplus \mathbb{C}$. Put $S=N_{1}=N_{2}=M_{1}=M_{2}=M_{3}=L=0$. Let $Q_{3}=E_{3}$, $Q_{1}=\{x(1,0,1 / 2) \in H \mid x \in \mathbb{C}\}$ and $Q_{2}=\{x(0,1,1 / 2) \in H \mid x \in \mathbb{C}\}$ This gives a Brenner type decomposition. We have another Brenner type decompositin by $Q_{3}^{\prime}=E_{3}, Q_{1}^{\prime}=\{x(1,0,1 / 3) \in H \mid x \in \mathbb{C}\}$ and $Q_{2}^{\prime}=\{x(0,1,2 / 3) \in H \mid x \in \mathbb{C}\}$ and the others are the same as the first one. Since $Q:=Q_{1}+Q_{2} \neq Q^{\prime}:=Q_{1}^{\prime}+Q_{2}^{\prime}$, they provide two kinds of Brenner type decompositions.

Let $\mathcal{S}=\left(H ; E_{1}, E_{2}, E_{3}\right)$ be a system of three subspaces which has a Brenner type decomposition. Then it is clear that for any $i, j, k=1,2,3$ with $i \neq j, j \neq k$ and $k \neq i$,

$$
\overline{E_{i}+E_{j}}+E_{k} \text { and }\left(E_{i} \cap E_{j}\right)+E_{k}
$$

are closed in $H$. We shall show that this topological property characterize a system of three subspaces which have a Brenner type decomposition.

Example 7. Let $\mathcal{S}=\left(H ; E_{1}, E_{2}, E_{3}\right)$ be a system of three subspaces in a Hilbert space $H$. If $\mathcal{S}=\left(H ; E_{1}, E_{2}, E_{3}\right)$ forms a pentagon (with $\left.E_{1} \supset E_{2}\right)$, then neither $E_{1}+E_{3}$ nor $E_{2}+E_{3}$ are closed. Therefore $\overline{E_{1}+E_{2}}+E_{3}=E_{1}+E_{2}$ is not closed and $\left(E_{1} \cap E_{2}\right)+E_{3}=E_{2}+E_{3}$ is not closed. Hence $\mathcal{S}=\left(H ; E_{1}, E_{2}, E_{3}\right)$ does not have a Brenner type decomposition. Thus this closedness property excludes pentagons to have a Brenner type decomposition.

We shall split out a distributive part and a double triangle part step by step.

Lemma 4.6. Let $\mathcal{S}=\left(H ; E_{1}, E_{2}, E_{3}\right)$ be a system of three subspaces in a Hilbert space $H$. Suppose that $\left(E_{1} \cap E_{2}\right)+E_{3}$ is closed. Then there exist systems $\mathcal{S}^{\prime}=\left(H^{\prime} ; E_{1}^{\prime}, E_{2}^{\prime}, E_{3}^{\prime}\right)$ and $\mathcal{S}^{\prime \prime}=\left(H^{\prime \prime} ; E_{1}^{\prime \prime}, E_{2}^{\prime \prime}, E_{3}^{\prime \prime}\right)$ of three subspaces such that

(1) $\mathcal{S}=\left(H ; E_{1}, E_{2}, E_{3}\right) \cong\left(H^{\prime} ; E_{1}^{\prime}, E_{2}^{\prime}, E_{3}^{\prime}\right) \oplus\left(H^{\prime \prime} ; E_{1}^{\prime \prime}, E_{2}^{\prime \prime}, E_{3}^{\prime \prime}\right)$.

(2) $E_{1}^{\prime \prime} \cap E_{2}^{\prime \prime}=0$

(3) $E_{3}^{\prime} \subset E_{1}^{\prime}=E_{2}^{\prime} \quad$ ("distributive component")

Proof. Consider two subspace decomposition for $F:=E_{1} \cap E_{2}$ and $E_{3}$. Since $\left(E_{1} \cap E_{2}\right)+E_{3}$ is closed, we may and do assume that there exits no angle part for $F:=E_{1} \cap E_{2}$ and $E_{3}$ up to isomorphism. Therefore we have the following decomposition:

$$
\begin{aligned}
& H=\left(E_{1} \cap E_{2}\right) \cap E_{3} \oplus\left(E_{1} \cap E_{2}\right) \cap E_{3}^{\perp} \oplus\left(E_{1} \cap E_{2}\right)^{\perp} \cap E_{3} \oplus\left(E_{1} \cap E_{2}\right)^{\perp} \cap E_{3}^{\perp} \\
& E_{1} \cap E_{2}=\left(E_{1} \cap E_{2} \cap E_{3}\right) \oplus\left(E_{1} \cap E_{2}\right) \cap E_{3}^{\perp} \oplus \quad 0 \quad \oplus \quad 0 \\
& E_{3}=\left(E_{1} \cap E_{2} \cap E_{3}\right) \oplus \quad 0 \quad \oplus\left(E_{1} \cap E_{2}\right)^{\perp} \cap E_{3} \oplus \quad 0
\end{aligned}
$$


Then put $H^{\prime}:=E_{1} \cap E_{2}$ and $H^{\prime \prime}:=\left(E_{1} \cap E_{2}\right)^{\perp}$. Consider corresponding decompositions for $H=H^{\prime} \oplus H^{\prime \prime}$. By taking intersections with $H^{\prime}$ and $H^{\prime \prime}$, let

$$
E_{1}^{\prime}=E_{1} \cap\left(E_{1} \cap E_{2}\right)=E_{1} \cap E_{2}, \quad E_{2}^{\prime}=E_{2} \cap\left(E_{1} \cap E_{2}\right)=E_{1} \cap E_{2},
$$

and

$$
\begin{aligned}
E_{3}^{\prime}=E_{3} & \cap\left(E_{1} \cap E_{2}\right)=E_{1} \cap E_{2} \cap E_{3} \\
E_{1}^{\prime \prime} & =E_{1} \cap\left(E_{1} \cap E_{2}\right)^{\perp}, \\
E_{2}^{\prime \prime} & =E_{2} \cap\left(E_{1} \cap E_{2}\right)^{\perp}, \\
E_{3}^{\prime \prime} & =E_{3} \cap\left(E_{1} \cap E_{2}\right)^{\perp}
\end{aligned}
$$

Then clearly we have that

$$
E_{1}^{\prime}+E_{1}^{\prime \prime}=E_{1} \quad E_{2}^{\prime}+E_{2}^{\prime \prime}=E_{2} \text { and } E_{3}^{\prime}+E_{3}^{\prime \prime}=E_{3}
$$

Moreover we have that $E_{3}^{\prime} \subset E_{1}^{\prime}=E_{2}^{\prime}$ and $E_{1}^{\prime \prime} \cap E_{2}^{\prime \prime}=0$.

Lemma 4.7. Let $\mathcal{S}=\left(H ; E_{1}, E_{2}, E_{3}\right)$ be a system of three subspaces in a Hilbert space $H$. Suppose that $\left(E_{1} \vee E_{2}\right)+E_{3}$ is closed. Then there exist systems $\mathcal{S}^{\prime}=\left(H^{\prime} ; E_{1}^{\prime}, E_{2}^{\prime}, E_{3}^{\prime}\right)$ and $\mathcal{S}^{\prime \prime}=\left(H^{\prime \prime} ; E_{1}^{\prime \prime}, E_{2}^{\prime \prime}, E_{3}^{\prime \prime}\right)$ of three subspaces such that

(1) $\mathcal{S}=\left(H ; E_{1}, E_{2}, E_{3}\right) \cong\left(H^{\prime} ; E_{1}^{\prime}, E_{2}^{\prime}, E_{3}^{\prime}\right) \oplus\left(H^{\prime \prime} ; E_{1}^{\prime \prime}, E_{2}^{\prime \prime}, E_{3}^{\prime \prime}\right)$.

(2) $E_{1}^{\prime \prime} \vee E_{2}^{\prime \prime}=H^{\prime \prime}$

(3) $E_{1}^{\prime}=E_{2}^{\prime}=0 \subset E_{3}^{\prime} \quad$ ("distributive component")

Proof. Consider two subspace decomposition for $F:=E_{1} \vee E_{2}$ and $E_{3}$. Since $\left(E_{1} \vee E_{2}\right)+E_{3}$ is closed, we may and do assume that there exits no angle part for $F:=E_{1} \vee E_{2}$ and $E_{3}$ up to isomorphism. Therefore we have the following decomposition:

$$
\begin{aligned}
H & =\left(E_{1} \vee E_{2}\right) \cap E_{3} \oplus\left(E_{1} \vee E_{2}\right) \cap E_{3}^{\perp} \oplus\left(E_{1} \vee E_{2}\right)^{\perp} \cap E_{3} \oplus\left(E_{1} \vee E_{2}\right)^{\perp} \cap E_{3}^{\perp} \\
E_{1} \vee E_{2} & =\left(E_{1} \vee E_{2}\right) \cap E_{3} \oplus\left(E_{1} \vee E_{2}\right) \cap E_{3}^{\perp} \oplus \quad 0 \quad 0 \\
E_{3} & =\left(E_{1} \vee E_{2}\right) \cap E_{3} \oplus \quad 0 \quad 0 \quad 0\left(E_{1} \vee E_{2}\right)^{\perp} \cap E_{3} \oplus \quad 0
\end{aligned}
$$

Then put $H^{\prime}:=\left(E_{1} \vee E_{2}\right)^{\perp}$ and $H^{\prime \prime}:=\left(E_{1} \vee E_{2}\right)$. Consider corresponding decompositions for $H=H^{\prime} \oplus H^{\prime \prime}$. By taking intersections with $H^{\prime}$ and $H^{\prime \prime}$, let

$$
\begin{aligned}
E_{1}^{\prime}=\left(E_{1} \vee E_{2}\right)^{\perp} E_{1}=0, & E_{2}^{\prime}=\left(E_{1} \vee E_{2}\right)^{\perp} E_{2}=0, \quad E_{3}^{\prime}=\left(E_{1} \vee E_{2}\right)^{\perp} \cap E_{3} \\
E_{1}^{\prime \prime} & =\left(E_{1} \vee E_{2}\right) \cap E_{1}=E_{1}, \\
E_{2}^{\prime \prime} & =\left(E_{1} \vee E_{2}\right) \cap E_{2}=E_{2}, \\
& E_{3}^{\prime \prime}=\left(E_{1} \vee E_{2}\right) \cap E_{3},
\end{aligned}
$$

Then clearly we have that

$$
E_{1}^{\prime}+E_{1}^{\prime \prime}=E_{1} \quad E_{2}^{\prime}+E_{2}^{\prime \prime}=E_{2} \quad \text { and } E_{3}^{\prime}+E_{3}^{\prime \prime}=E_{3}
$$


Moreover we have that $E_{1}^{\prime}=E_{2}^{\prime}=0 \subset E_{3}^{\prime}$ and $E_{1}^{\prime \prime} \vee E_{2}^{\prime \prime}=H^{\prime \prime}$.

Lemma 4.8. Let $H$ be a Hilbert space and $H_{1}$ and $H_{2}$ be two closed subspaces of $H$. Assume that $H=H_{1}+H_{2}$ and $H_{1} \cap H_{2}=0$. Let $F_{i}$ be an algebraic linear subspace of $H_{i}$ for $i=1,2$. Consider their algebraic linear sum $F:=F_{1}+F_{2}$ in $H$. Then $F$ is closed if and only if $F_{1}$ and $F_{2}$ are closed.

Proof. There exists a closed subspace $M \subset H$ such that $\left(H ; H_{1}, H_{2}\right)$ is isomorphic to $\left(H ; M, M^{\perp}\right)$. Then the statement is redeuced to this case where the statement is clear.

By the Lemma above, we have immediately have the followings:

Lemma 4.9. Let $\mathcal{S}=\left(H ; E_{1}, E_{2}, E_{3}\right)$ be a system of three subspaces in a Hilbert space $H$. Suppose that there exist systems $\mathcal{S}^{\prime}=\left(H^{\prime} ; E_{1}^{\prime}, E_{2}^{\prime}, E_{3}^{\prime}\right)$ and $\mathcal{S}^{\prime \prime}=\left(H^{\prime \prime} ; E_{1}^{\prime \prime}, E_{2}^{\prime \prime}, E_{3}^{\prime \prime}\right)$ of three subspaces such that

$$
\mathcal{S}=\left(H ; E_{1}, E_{2}, E_{3}\right) \cong\left(H^{\prime} ; E_{1}^{\prime}, E_{2}^{\prime}, E_{3}^{\prime}\right) \oplus\left(H^{\prime \prime} ; E_{1}^{\prime \prime}, E_{2}^{\prime \prime}, E_{3}^{\prime \prime}\right) .
$$

Then the followings hold:

(1) $\left(E_{1} \cap E_{2}\right)+E_{3}$ is closed in $H$ if and only if $\left(E_{1}^{\prime} \cap E_{2}^{\prime}\right)+E_{3}^{\prime}$ is closed in $H^{\prime}$ and $\left(E_{1}^{\prime \prime} \cap E_{2}^{\prime \prime}\right)+E_{3}^{\prime \prime}$ is closed in $H^{\prime \prime}$.

(2) $\left(E_{1} \vee E_{2}\right)+E_{3}$ is closed in $H$ if and only if $\left(E_{1}^{\prime} \vee E_{2}^{\prime}\right)+E_{3}$ is closed in $H^{\prime}$ and $\left(E_{1}^{\prime} \vee E_{2}^{\prime}\right)+E_{3}^{\prime}$ is closed in $H^{\prime \prime}$.

(3) $E_{1} \cap E_{2}=0$ if and only if $E_{1}^{\prime} \cap E_{2}^{\prime}=0$ and $E_{1}^{\prime \prime} \cap E_{2}^{\prime \prime}=0$.

(4) $E_{1} \vee E_{2}=H$ if and only if $E_{1}^{\prime} \vee E_{2}^{\prime}=H^{\prime}$ and $E_{1}^{\prime \prime} \vee E_{2}^{\prime \prime}=H^{\prime \prime}$

Theorem 4.10. Let $\mathcal{S}=\left(H ; E_{1}, E_{2}, E_{3}\right)$ be a system of three subspaces in a Hilbert space $H$. Then the followings are equivalent:

(1) Linear sums $\left(E_{i} \vee E_{j}\right)+E_{k}$ and $\left(E_{i} \cap E_{j}\right)+E_{k}$ are closed for $i, j, k \in\{1,2,3\}$ with $i \neq j \neq k \neq i$.

(2) $\mathcal{S}$ has a Brenner type decomposition.

Proof. It is trival that (2) implies (1). Conversely, assume (1). Since $\left(E_{1} \cap E_{2}\right)+E_{3}$ is closed, there exist systems $\mathcal{S}^{\prime}=\left(H^{\prime} ; E_{1}^{\prime}, E_{2}^{\prime}, E_{3}^{\prime}\right)$ and $\mathcal{S}^{\prime \prime}=\left(H^{\prime \prime} ; E_{1}^{\prime \prime}, E_{2}^{\prime \prime}, E_{3}^{\prime \prime}\right)$ of three subspaces such that

(1) $\mathcal{S}=\left(H ; E_{1}, E_{2}, E_{3}\right) \cong\left(H^{\prime} ; E_{1}^{\prime}, E_{2}^{\prime}, E_{3}^{\prime}\right) \oplus\left(H^{\prime \prime} ; E_{1}^{\prime \prime}, E_{2}^{\prime \prime}, E_{3}^{\prime \prime}\right)$.

(2) $E_{1}^{\prime \prime} \cap E_{2}^{\prime \prime}=0$

(3) $E_{3}^{\prime} \subset E_{1}^{\prime}=E_{2}^{\prime} \quad$ ("distributive component")

Since $\left(E_{1}^{\prime \prime} \vee E_{2}^{\prime \prime}\right)+E_{3}^{\prime \prime}$ is closed. Then there exist systems $\mathcal{S}^{\prime \prime \prime}=$ $\left(H^{\prime \prime \prime} ; E_{1}^{\prime \prime \prime}, E_{2}^{\prime \prime \prime}, E_{3}^{\prime \prime \prime}\right)$ and $\mathcal{S}^{\prime \prime \prime \prime}=\left(H^{\prime \prime \prime \prime} ; E_{1}^{\prime \prime \prime \prime}, E_{2}^{\prime \prime \prime \prime}, E_{3}^{\prime \prime \prime \prime}\right)$ of three subspaces such that

(1) $\mathcal{S}^{\prime \prime}=\left(H^{\prime \prime} ; E_{1}^{\prime \prime}, E_{2}^{\prime \prime}, E_{3}^{\prime \prime}\right) \cong\left(H^{\prime \prime \prime} ; E_{1}^{\prime \prime \prime}, E_{2}^{\prime \prime \prime}, E_{3}^{\prime \prime \prime}\right) \oplus\left(H^{\prime \prime \prime \prime} ; E_{1}^{\prime \prime \prime \prime}, E_{2}^{\prime \prime \prime \prime}, E_{3}^{\prime \prime \prime \prime}\right)$.

(2) $E_{1}^{\prime \prime \prime \prime} \vee E_{2}^{\prime \prime \prime \prime}=H^{\prime \prime \prime \prime}$

(3) $E_{1}^{\prime \prime \prime}=E_{2}^{\prime \prime \prime}=0 \subset E_{3}^{\prime \prime \prime} \quad$ ("distributive component ") 
Therefore

$$
\begin{aligned}
\mathcal{S} & =\left(H ; E_{1}, E_{2}, E_{3}\right) \\
& \cong\left(H^{\prime} ; E_{1}^{\prime}, E_{2}^{\prime}, E_{3}^{\prime}\right) \oplus\left(H^{\prime \prime \prime} ; E_{1}^{\prime \prime \prime}, E_{2}^{\prime \prime \prime}, E_{3}^{\prime \prime \prime}\right) \oplus\left(H^{\prime \prime \prime \prime} ; E_{1}^{\prime \prime \prime \prime}, E_{2}^{\prime \prime \prime \prime}, E_{3}^{\prime \prime \prime \prime}\right)
\end{aligned}
$$

Moreover $E_{1}^{\prime \prime \prime \prime} \vee E_{2}^{\prime \prime \prime \prime}=H^{\prime \prime \prime \prime}$ and $E_{1}^{\prime \prime \prime \prime} \cap E_{2}^{\prime \prime \prime \prime}=0$. We shall split out a distributive part and a double triangle part step by step using Lemma 4.6. Lemma 4.7, Lemma 4.8 and Lemma 4.9, Since closedness property is preserved after we split out one step, we can proceed the next step.

Finally we can split out a double triangle part $\left(Q ; Q_{1}, Q_{2}, Q_{3}\right)$, because in the final step we have that

$$
Q_{i} \vee Q_{j}=Q, \quad \text { and } \quad Q_{i} \wedge Q_{j}=0, \quad(i \neq j, i, j=1,2,3) .
$$

and the rest part consists of finite direct sum of distributive systems of three subspaces. Hence we have (2).

\section{DENSE DECOMPOSITION}

In an infinite-dimensional Hilbert space $H$, the algebraic linear sum $H^{\prime}+H^{\prime \prime}$ of closed subspaces $H^{\prime}$ and $H^{\prime \prime}$ is not necessarily closed. Therefore we cannot expect direct sum decomposition in general.

Example 8. Let $\mathcal{S}=\left(H ; E_{1}, E_{2}, E_{3}\right)$ be a system of three subspaces in a Hilbert space $H$. Suppose that $E_{1} \cap E_{2}=0$ and $E_{1}+E_{2}$ is not closed and $E_{1} \vee E_{2}=\overline{E_{1}+E_{2}}=E_{3}=H$. Put $H^{\prime}=E_{1}$ and $H^{\prime \prime}=E_{2}$. Then $H$ has a "dense decomposition" $H=\overline{H^{\prime}+H^{\prime \prime}}$ such that $H^{\prime} \cap H^{\prime \prime}=0$. $E_{1}=E_{1}+0, E_{2}=0+E_{2}$ and $E_{3}=\overline{E_{1}+E_{2}}$

Definition. Let $\mathcal{S}=\left(H ; E_{1}, E_{2}, E_{3}\right)$ be a system of three subspaces in a Hilbert space $H$ and let $\mathcal{S}^{(k)}=\left(H^{(k)} ; E_{1}^{(k)}, E_{2}^{(k)}, E_{3}^{(k)}\right)$ be systems of three subspaces in a Hilbert space $H^{(k)}$ for $k=1,2, \ldots, m$. Then $\mathcal{S}$ is said to have a dense decomposition

$$
\mathcal{S} \supset \mathcal{S}^{(1)} \oplus_{\text {alg }} \cdots \oplus_{\text {alg }} \mathcal{S}^{(m)} \quad(\text { dense })
$$

if $H=\overline{H^{(1)} \oplus_{\text {alg }} \cdots \oplus_{\text {alg }} H^{(m)}}, E_{1}=\overline{E_{1}^{(1)} \oplus_{\text {alg }} \cdots \oplus_{\text {alg }} E_{1}^{(m)}}$,

$E_{2}=\overline{E_{2}^{(1)} \oplus_{\text {alg }} \cdots \oplus_{\text {alg }} E_{2}^{(m)}}$ and $E_{3}=\overline{E_{3}^{(1)} \oplus_{\text {alg }} \cdots \oplus_{\text {alg }} E_{3}^{(m)}}$. In particular, $H^{(1)}, \ldots H^{(m)}$ are linearly independent.

In geneal, we define a dense decomposition of a system of $n$-subspaces similarly.

For example,any system of two subspaces $\mathcal{S}=\left(H ; E_{1}, E_{2}\right)$ has a dense decomposition satisfying distributive law. In fact,

$\left(E_{1} \cap E_{2}\right) \oplus_{a l g}(K \oplus O) \oplus_{a l g} \operatorname{Im}\left(\begin{array}{cc}c^{2} & c s \\ c s & s^{2}\end{array}\right) \oplus_{\text {alg }}\left(E_{1} \cap E_{2}^{\perp}\right) \oplus_{\text {alg }}\left(E_{1}^{\perp} \cap E_{2}\right) \oplus_{\text {alg }}\left(E_{1}^{\perp} \cap E_{2}^{\perp}\right)$.

is a dense decomposition of $H$. We expect that a certain class of systems $\mathcal{S}$ of three subspaces has a dense decomposition with a distributive part 
$H^{d i s}$, a double triangle part $Q$ and six kinds of pentagon parts

$$
H^{\sigma}=\overline{\sum_{\sigma \in S_{3}}\left(E_{\sigma(1)}^{\sigma} \oplus_{a l g} E_{\sigma(3)}^{\sigma}\right)}
$$

(with $E_{\sigma(3)}^{\sigma} \supset E_{\sigma(2)}^{\sigma}$ ), for a permutation $\sigma \in S_{3}$ on three letters $\{1,2,3\}$.

A distributive part is an algebraic sum of $2^{3}=8$ components

$$
H^{d i s}=S \oplus_{\text {alg }} N_{1} \oplus_{\text {alg }} N_{2} \oplus_{\text {alg }} N_{3} \oplus_{\text {alg }} M_{1} \oplus_{\text {alg }} M_{2} \oplus_{\text {alg }} M_{3} \oplus_{\text {alg }} L
$$

and a double triangle part is a Hilbert space $Q$ with

$$
Q=\overline{Q_{1} \oplus_{\text {alg }} Q_{2}}=\overline{Q_{2} \oplus_{\text {alg }} Q_{3}}=\overline{Q_{3} \oplus_{\text {alg }} Q_{1}} .
$$

Then $\left(H^{\sigma} ; E_{1}^{\sigma}, E_{2}^{\sigma}, E_{3}^{\sigma}\right)$ form pentagons (with $\left.E_{\sigma(3)}^{\sigma} \supset E_{\sigma(2)}^{\sigma}\right)$, so that $E_{\sigma(1)}^{\sigma} \vee E_{\sigma(2)}^{\sigma}=H^{\sigma}, E_{\sigma(1)}^{\sigma} \wedge E_{\sigma(3)}^{\sigma}=0$, and $E_{\sigma(3)}^{\sigma} \supset E_{\sigma(2)}^{\sigma}$ with $E_{\sigma(3)}^{\sigma} \neq E_{\sigma(2)}^{\sigma}$.

In this way

$$
S \oplus_{\text {alg }} N_{1} \oplus_{\text {alg }} N_{2} \oplus_{\text {alg }} N_{3} \oplus_{\text {alg }} M_{1} \oplus_{\text {alg }} M_{2} \oplus_{\text {alg }} M_{3} \oplus_{\text {alg }} L \oplus_{\text {alg }} Q \oplus_{\text {alg }} \sum_{\sigma \in S_{3}}\left(E_{\sigma(1)}^{\sigma} \oplus_{\text {alg }} E_{\sigma(3)}^{\sigma}\right)
$$

is dense in $H$.

But we do not know whether this kinds of decompostion hold or not in general.

Finally we give a partial result which gives a condition on a system to have a (dense) decomposition containing a pentagon.

Lemma 5.1. Let $\mathcal{S}=\left(H ; E_{1}, E_{2}, E_{3}\right)$ be a system of three subspaces in a Hilbert space $H$. Suppose that there exist systems $\mathcal{S}^{\prime}=\left(H^{\prime} ; E_{1}^{\prime}, E_{2}^{\prime}, E_{3}^{\prime}\right)$ and $\mathcal{S}^{\prime \prime}=\left(H^{\prime \prime} ; E_{1}^{\prime \prime}, E_{2}^{\prime \prime}, E_{3}^{\prime \prime}\right)$ of three subspaces such that

(1) $\mathcal{S}=\left(H ; E_{1}, E_{2}, E_{3}\right) \cong\left(H^{\prime} ; E_{1}^{\prime}, E_{2}^{\prime}, E_{3}^{\prime}\right) \oplus\left(H^{\prime \prime} ; E_{1}^{\prime \prime}, E_{2}^{\prime \prime}, E_{3}^{\prime \prime}\right)$.

(2) $\left(H^{\prime} ; E_{1}^{\prime}, E_{2}^{\prime}, E_{3}^{\prime}\right)$ forms a pentagon (with $\left.E_{3}^{\prime} \supset E_{2}^{\prime}\right)$.

(3) (distributive component) there exist subspaces $N_{1}, N_{2}$ and $M_{1}$ of $H^{\prime \prime}$ such that

$$
\begin{gathered}
H^{\prime \prime}=N_{1} \oplus N_{2} \oplus M_{1}, \\
E_{1}^{\prime \prime}=0 \oplus N_{2} \oplus M_{1}, E_{2}^{\prime \prime}=N_{1} \oplus 0 \oplus 0 \text { and } E_{3}^{\prime \prime}=N_{1} \oplus N_{2} \oplus 0 .
\end{gathered}
$$

Then $E_{1} \wedge E_{2}=0, E_{1} \vee E_{2}=H$ and $E_{2} \varsubsetneqq E_{3}$.

Proof. It is clear.

We can rearrange the above decomposition such that $\left(H^{\prime} ; E_{1}^{\prime} \oplus M_{1}, E_{2}^{\prime} \oplus\right.$ $\left.N_{1}, E_{3}^{\prime} \oplus N_{1}\right)$ forms a pentagon (with $\left.E_{3}^{\prime} \oplus N_{1} \supset E_{2}^{\prime} \oplus N_{1}\right)$ and $\left(N_{2} ; N_{2}, 0, N_{2}\right.$ ) is a distributive part.

We shall show that the converse of the Lemma above holds in the sense of dense decomposition.

Proposition 5.2. Let $\mathcal{S}=\left(H ; E_{1}, E_{2}, E_{3}\right)$ be a system of three subspaces in a Hilbert space $H$. Suppose that

$$
E_{1} \wedge E_{2}=0, E_{1} \vee E_{2}=H \text { and } E_{2} \varsubsetneqq E_{3} .
$$


We assume that $E_{3} / E_{2}$ is finite dimensional. Then we have the following:

(i) If $E_{3} \neq E_{3} \cap\left(E_{1}+E_{2}\right)$, then there exist subspaces $E_{1}^{\prime}, E_{2}^{\prime}, E_{3}^{\prime}$ and $N_{2}$ of $H$ such that

(1) $H \supset E_{1}^{\prime} \oplus_{\text {alg }} E_{3}^{\prime} \oplus_{\text {alg }} N_{2}$ (dense)

(2) $\left(E_{1}^{\prime} \vee E_{3}^{\prime} ; E_{1}^{\prime}, E_{2}^{\prime}, E_{3}^{\prime}\right)$ forms a pentagon (with $\left.E_{3}^{\prime} \supset E_{2}^{\prime}\right)$.

(3) $E_{1}=\overline{E_{1}^{\prime} \oplus_{\text {alg }} N_{2}}, E_{2}=E_{2}^{\prime}$ and $E_{3}=\overline{E_{3}^{\prime} \oplus_{\text {alg }} N_{2}}$

Moreover $\left(N_{2} ; N_{2}, 0, N_{2}\right)$ is a distributive part.

(ii) If $E_{3}=E_{3} \cap\left(E_{1}+E_{2}\right)$, then there exist subspaces $N_{1}, N_{2}$ and $M_{1}$ of $H$ such that

$$
H \supset N_{1} \oplus_{\text {alg }} N_{2} \oplus_{\text {alg }} M_{1} \text { (dense) , }
$$

$E_{1}=0+N_{2}+M_{1}, E_{2}=N_{1}+0+0$ and $E_{3}=N_{1}+N_{2}+0$,

Proof. Case (i): Assume that $E_{3} \neq E_{3} \cap\left(E_{1}+E_{2}\right)$.

Put $F_{3}=E_{3} \cap\left(E_{3} \cap\left(E_{1}+E_{2}\right)\right)^{\perp} \neq 0$. Then $E_{3}=\left(E_{3} \cap\left(E_{1}+E_{2}\right)\right) \oplus F_{3}$. Since $E_{3} \supset E_{2}, F_{3}$ is orthgonal to $E_{2}$. We shall show that

$$
E_{1} \cap\left(E_{2}+F_{3}\right)=0
$$

In fact, let $x_{1}=x_{2}+f_{3} \in E_{1} \cap\left(E_{2}+F_{3}\right)$ for $x_{1} \in E_{1}, x_{2} \in E_{2}$ and $f_{3} \in F_{3}$. Then $f_{3}=x_{1}-x_{2} \in E_{3} \cap\left(E_{1}+E_{2}\right)$. But $f_{3}$ is also in $\left(E_{3} \cap\left(E_{1}+E_{2}\right)\right)^{\perp}$. Hence $f_{3}=0$. Then $x_{1}=x_{2}$ is in $E_{1} \cap E_{2}=0$. Therefore $x_{1}=x_{2}=0$.

Since $E_{3} / E_{2}$ is finite dimensional, we can find

$$
u_{1}, \ldots, u_{n} \in E_{3} \cap\left(E_{1}+E_{2}\right)\left(\supset E_{2}\right)
$$

such that the quotient image $\overline{u_{1}}, \ldots, \overline{u_{n}}$ are linearly independent in the quotient $E_{3} / E_{2}$ and

$$
E_{3} \cap\left(E_{1}+E_{2}\right)=E_{2}+\left[u_{1}, \ldots, u_{n}\right],
$$

where $\left[u_{1}, \ldots, u_{n}\right]$ is a linear span of $u_{1}, \ldots, u_{n}$. In particular, $E_{3} \cap$ $\left(E_{1}+E_{2}\right)$ is closed in $H$. Choose $v_{1}, \ldots, v_{n} \in E_{1}$ and $w_{1}, \ldots, w_{n} \in E_{2}$ such that

$$
u_{k}=v_{k}+w_{k} \quad(k=1, \ldots, n) .
$$

Since the quotient image $\overline{u_{k}}=\overline{v_{k}}$ for $k=1, \ldots, n$, we have that $v_{1}, \ldots, v_{n}$ are linearly independent.

Put $N_{2}=\left[v_{1}, \ldots, v_{n}\right]$. Since $E_{2} \subset E_{3}, v_{k}=u_{k}-w_{k}$ is also in $E_{3}$. Hence $N_{2} \subset E_{1} \cap E_{3}$. Since $w_{1}, \ldots, w_{n}$ are in $E_{2}$, we have that

$$
E_{3} \cap\left(E_{1}+E_{2}\right)=E_{2}+\left[v_{1}, \ldots, v_{n}\right],
$$

Put $E_{1}^{\prime}=E_{1} \cap\left[v_{1}, \ldots, v_{n}\right]^{\perp}, E_{2}^{\prime}=E_{2}$ and $E_{3}^{\prime}=E_{2} \oplus F_{3}$. Then

$$
E_{3}=\left(E_{3} \cap\left(E_{1}+E_{2}\right)\right) \oplus F_{3}=\left(E_{2}+\left[v_{1}, \ldots, v_{n}\right]\right) \oplus F_{3} .
$$


We shall show that $N_{2}, E_{1}^{\prime}, E_{2}$ and $F_{3}$ are linearly independent. In fact, let $n_{2}+x_{1}+x_{2}+f_{3}=0$ for $n_{2} \in N_{2}, x_{1} \in E_{1}^{\prime}, x_{2} \in E_{2}$ and $f_{3} \in F_{3}$. Then

$$
n_{2}+x_{1}=-x_{2}-f_{3} \in E_{1} \cap\left(E_{2}+F_{3}\right)=0 .
$$

Therefore $n_{2}+x_{1}=0$ and $x_{2}+f_{3}=0$. Since $N_{2} \perp E_{1}^{\prime}$ and $F_{3} \perp E_{2}$, we have that $n_{2}=x_{1}=x_{2}=f_{3}=0$. Since

$$
E_{1}^{\prime} \cap E_{3}^{\prime}=E_{1}^{\prime} \cap\left(E_{2}^{\prime}+F_{3}\right) \subset E_{1} \cap\left(E_{2}+F_{3}\right)=0,
$$

$\left(E_{1}^{\prime} \vee E_{3}^{\prime} ; E_{1}^{\prime}, E_{2}^{\prime}, E_{3}^{\prime}\right)$ forms a pentagon (with $\left.E_{3}^{\prime} \supset E_{2}^{\prime}\right)$. And $E_{1}=$ $\overline{E_{1}^{\prime} \oplus_{\text {alg }} N_{2}}, E_{2}=E_{2}^{\prime}$ and $E_{3}=\overline{E_{3}^{\prime} \oplus_{\text {alg }} N_{2}}$.

Case (ii): Assume that $E_{3}=E_{3} \cap\left(E_{1}+E_{2}\right)$. Then $F_{3}=E_{3} \cap\left(E_{3} \cap\right.$ $\left.\left(E_{1}+E_{2}\right)\right)^{\perp}=0$. We can similarly define $u_{k}, v_{k}, w_{k}$ as above. Put $N_{2}=\left[v_{1}, \ldots, v_{n}\right]$. Define $M_{1}=E_{1} \cap\left[v_{1}, \ldots, v_{n}\right]^{\perp}, N_{1}=E_{2}$. Then $E_{1}=N_{2} \oplus M_{1}$ and $E_{3}=N_{1}+N_{2}$. Moreover $N_{1}+N_{2}+M_{1}=E_{2}+E_{1}$ is dense in $H$. And $N_{1}, N_{2}$ and $M_{1}$ are linearly independent. In fact, let $n_{1}+n_{2}+m_{1}=0$ for $n_{1} \in N_{1}, n_{2} \in N_{2}$ and $m_{1} \in M_{1}$. Then $n_{2}+m_{1}=-n_{1} \in E_{1} \cap E_{2}=0$. Hence $n_{2}+m_{1}=n_{1}=0$. Since $N_{2}$ and $M_{1}$ are orthogonal, $n_{2}=m_{1}=0$. Hence

$$
H \supset N_{1} \oplus_{\text {alg }} N_{2} \oplus_{\text {alg }} M_{1} \text { (dense), }
$$

Example 9.Let $K=\ell^{2}(\mathbb{N})$ be the Hilbert space of square summable sequences. Let $A: K \rightarrow K$ be a diagonal operator such that $(A x)_{n}=\frac{1}{n} x_{n}$ for $x=\left(x_{n}\right)_{n} \in K$. Then $\operatorname{Im} A$ is dense in $K$ and not equal to $K$. Put $f=(1,1 / 2,1 / 3, \ldots, 1 / n, \ldots) \in K$ and $v=$ $(0,1 / 2,1 / 3, \ldots, 1 / n, \ldots) \in K$. Then $f$ and $v$ are not in $\operatorname{Im} A$. Define $N_{2}=\mathbb{C}(0, v), H=K \oplus K, E_{1}=(K \oplus 0)+N_{2}, E_{2}=\{(x, A x) \mid x \in K\}$ and $E_{3}=E_{2}+\mathbb{C}(0, f)+N_{2}$. Put $E_{1}^{\prime}=K \oplus 0, E_{2}^{\prime}=\{(x, A x) \mid x \in K\}$ and $E_{3}^{\prime}=E_{2}+\mathbb{C}(0, f)$. Then $\left(E_{1}^{\prime} \vee E_{3}^{\prime} ; E_{1}^{\prime}, E_{2}^{\prime}, E_{3}^{\prime}\right)$ forms a pentagon (with $E_{3}^{\prime} \supset E_{2}^{\prime}$ ).

\section{REFERENCES}

[1] H. Araki, A lattice of von Neumann algebras with the quantum theory of a free Bose field, J. Math. Phys. 4(1963), 1343-1362.

[2] A. Botter, I. Gohberg, Yu. I. Karlovich, N. Krupnik, S. Roch, B. Silbermann and I. Spittokovsky, Banach algebras generated by $N$ idempotents and Application, Singular Integral Operators and Related Topics, Birkhauser (1996), 19-54.

[3] S. Brenner, Endomorphism algebras of vector spaces with distinguished sets of subspaces, J. Algebra 6 (1967), 100-114.

[4] C. Davis, Separation of two linear suspaces, Acta Sci. Math. Szeged. 19 (1958), 172-187.

[5] J. Dixmier, Position relative de deux variétés linéaires fermées dans un espace de Hilbert, Rev. Sci. 86 (1948), 387-399.

[6] M. Enomoto and Y. Watatani, Relative position of four subspaces in a Hilbert space, Adv. Math. 201 (2006), 263-317. 
[7] M. Enomoto and Y. Watatani, Indecomposable representations of quivers on infinite-dimensional Hilbert spaces, J. Funct. Anal. 256 (2009), 959-991.

[8] I. S. Feshchenko, On closedness of the sum of $n$ subspaces of a Hilbert space, Ukrainian Math. J., 63 (2012), 1566-1622.

[9] P. Gabriel, Unzerlegbare Darstellungen I, Manuscripta Math. 6 (1972), 71-103.

[10] I. M. Gelfand and V. A. Ponomarev, Problems of linear algebra and classification of quadruples of subspaces in a finite-dimensional vector space, Coll. Math. Spc. Bolyai 5, Tihany (1970), 163-237.

[11] G. Graetzer, Lattice theory: Foundation, Birkhaeuser, 2011.

[12] L. Ge and W. Yuan, Kadison-Singer algebras, I: hyperfinite case, Proc. Natl. Acad. Sci. USA 107 (2010), 1838-1843.

[13] L. Ge and W. Yuan, Kadison-Singer algebras, II: general case, Proc. Natl. Acad. Sci. USA 107 (2010), 4840-4844.

[14] D. W. Hadwin, W. E. Longstaff and P. Rosenthal, Small transitive lattices, Proc. Amer. Math. Soc. 87 (1983), 121-124.

[15] P. R. Halmos, Two subspaces, Trans. Amer. Math. Soc. 144(1969), 381-389.

[16] P. R. Halmos, Ten problems in Hilbert space, Bull. Amer. Math. Soc. 76 (1970), 887-933.

[17] K.J. Harrison, H. Radjavi and P. Rosenthal, A transitive medial subspace lattice, Proc. Amer. Math. Soc. 28 (1971), 119-121.

[18] S. $\mathrm{Hu}$ and $\mathrm{Y}$. Xue, $C^{*}$-algebras generated by three projections, arXiv:1207.6890 math.OA]

[19] C.Jian and Z.X Wang, Strongly Irreducible Operators on Hilbert Space, Longman, 1998.

[20] V. Jones, Index for subfactors, Inv. Math. 72(1983), 1-25.

[21] S. Kruglyak, V. Rabanovich and Y. Samoilenko, On sums of projections, Functional Anal. Appl., 36(2002), 182-195.

[22] S. Kruglyak and Y. Samoilenko, On the complexity of description of representations of $*$-algebras generated by idempotents, Proc. Amer. Math. Soc., 128(2000), 1655-1664.

[23] Y. Moskaleva and Y. Samoilenko,Systems of $n$ subspaces and representations of *algebras generated by projections, Methods Funct. Anal. Topology 12 (2006), 57-73.

[24] V. S. Sunder, N-subspaces, Canad. J. Math. 40 (1988), 38-54.

(Masatoshi Enomoto) 1-6-13, Nogami, TAKarazuka,Hyogo, Japan

E-mail address: enomotoma@hotmail.co.jp

(Yasuo Watatani) Department of Mathematical Sciences, Kyushu UniVERSITY, HAKOZAKI, FUKUOKA, 812-8581, JAPAN

E-mail address: watatani@math.kyushu-u.ac.jp 